\title{
Phosphorylated-FAK Y397
}

National Cancer Institute

\section{Source}

National Cancer Institute. Phosphorylated-FAK Y397. NCI Thesaurus. Code C153558.

A post-translationally modified form of focal adhesion kinase 1 (FAK; FAK1; PT K2) where the tyrosine (Y; Tyr) at residue 397 is phosphorylated. Cell adhesion-signaling promotes the association of FAK with Src family kinases, which stimulates autophosphorylation of FAK Y397 and creates a SH2-binding site on FAK to promote Src family kinase- and integ rin-mediated signaling. 